\title{
Floquet Theory for Discontinuously Supported Waveguides
}

\author{
A. Sorzia \\ Dipartimento di Scienze e Metodi dell'Ingegneria (DISMI), Universitá degli Studi di Modena e Reggio Emilia, Via G. Amendola 2, \\ 42122 Reggio Emilia, Italy \\ Correspondence should be addressed to A. Sorzia; andrea.sorzia@unimore.it
}

Received 24 December 2015; Accepted 5 May 2016

Academic Editor: Julius Kaplunov

Copyright (c) 2016 A. Sorzia. This is an open access article distributed under the Creative Commons Attribution License, which permits unrestricted use, distribution, and reproduction in any medium, provided the original work is properly cited.

We apply Floquet theory of periodic coefficient second-order ODEs to an elastic waveguide. The waveguide is modeled as a uniform elastic string periodically supported by a discontinuous Winkler elastic foundation and, as a result, a Hill equation is found. The fundamental solutions, the stability regions, and the dispersion curves are determined and then plotted. An asymptotic approximation to the dispersion curve is also given. It is further shown that the end points of the band gap structure correspond to periodic and semiperiodic solutions of the Hill equation.

\section{Introduction}

Periodic structures often appear in several mechanical systems, ranging from strings and beams $[1,2]$ to phononic crystals [3-5] to name just a few. Such systems exhibit a typical pass/block band structure when wave propagation is considered. Indeed, periodic structures are especially relevant when employed as waveguides $[6,7]$ or energy scavenging devices [8]. The analysis of the transmission property of waveguides is best carried out through Floquet theory of periodic coefficient ODEs, although this fact is seldom neglected in favor of a more direct approach by means of the Floquet-Bloch boundary conditions. In this paper, an analysis of the mechanical problem of a uniform string periodically supported on a Winkler foundation is presented form the standpoint of the stability theory of Hill's equation [9]. Besides, a high-frequency asymptotic homogenization procedure is presented, following $[10,11]$. The discontinuous character of the support may be due to crack propagation [12-15] or debonding in composite materials [16, 17]. It could also be due to the tensionless character of the substrate [18]. This study follows upon a very vast body of literature on elastic periodic structures [2,19-24]. The situation of wave propagation through a thin coating layer [25-27] could also be considered. Applications in the realm of civil engineering are also possible [28-32]. The paper is structured as follows: Section 2 sets up the mechanical model and the governing equations. Section 3 discusses stability of the solution of Hill's equation. The dispersion relation and its asymptotic approximation are presented in Section 4. Finally, conclusions are drawn in Section 5.

\section{The Mechanical Model}

Let us consider a homogeneous elastic string in uniform tension $T$, periodically supported on a Winkler elastic foundation (Figure 1). The governing equation for the transverse displacement $w(x, t)$ in the absence of loading is periodic with period $L$ :

$$
\begin{aligned}
&-T \partial_{x x} w+\rho A \partial_{t t} w+(1-H) \beta w= 0, \\
& x \in\left(-L_{1}, L-L_{1}\right),
\end{aligned}
$$

where $\rho A$ is the mass linear density of the string, assumed constant, $\beta$ is the Winkler subgrade modulus (with physical dimension of stress), and $H$ is Heaviside step function; that is,

$$
H(x)= \begin{cases}1, & x>0, \\ 0, & x<0 .\end{cases}
$$

This equation may be rewritten in dimensionless form

$$
-\kappa^{2} w^{\prime \prime}+\ddot{w}+(1-H) w=0, \quad x_{1} \in(-\alpha, 1-\alpha),
$$




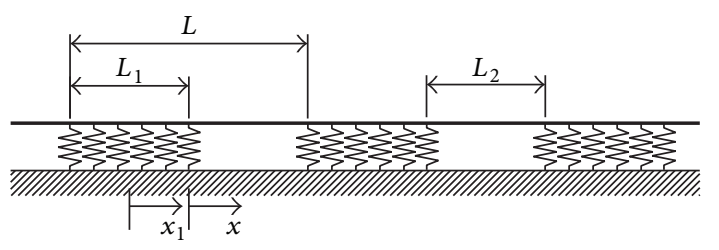

FIGURE 1: A homogeneous string periodically supported by an elastic foundation.

having introduced the dimensionless positive ratios:

$$
\begin{aligned}
& \kappa=\sqrt{\frac{T}{\beta L^{2}}}, \\
& \alpha=\frac{L_{1}}{L} \leq 1,
\end{aligned}
$$

together with $x_{1}=x / L \in[0,1]$, the dimensionless axial coordinate, and $\tau=t / \sqrt{\rho A / \beta}$, the dimensionless time. Here, prime denotes differentiation with respect to $x_{1}$ and dot differentiation with respect to $\tau$ and $w\left(x_{1}, \tau\right)$ and $H\left(x_{1}\right)$ are assumed. We shall look for the harmonic behavior of $w$; that is, $w\left(x_{1}, \tau\right)=u\left(x_{1}\right) \exp (i \Omega \tau)$, whence (3) becomes the constant coefficient ODE for $u\left(x_{1}\right)$ :

$$
-\kappa^{2} u^{\prime \prime}-\Omega^{2} u+(1-H) u=0, \quad x_{1} \in(-\alpha, 1-\alpha) .
$$

We shift the unit period to range in the interval $(-\alpha / 2,1-\alpha / 2)$ in order to consider an even/odd problem; namely,

$$
\begin{aligned}
-\kappa^{2} u^{\prime \prime}-\Omega^{2} u+\left[1-H\left(x_{1}-\frac{\alpha}{2}\right)\right] u & =0, \\
x_{1} & \in\left(-\frac{\alpha}{2}, 1-\frac{\alpha}{2}\right) .
\end{aligned}
$$

The general harmonic solution of (3) in the supported region $x_{1} \in(-\alpha / 2, \alpha / 2)$ is given by

$$
\begin{aligned}
u_{s}\left(x_{1}\right)= & A_{1} \exp \left(\frac{\sqrt{1-\Omega^{2}}}{\kappa} x_{1}\right) \\
& +A_{2} \exp \left(-\frac{\sqrt{1-\Omega^{2}}}{\kappa} x_{1}\right),
\end{aligned}
$$

while the solution in the free region $x_{1} \in(\alpha / 2,1-\alpha / 2)$ is

$$
u_{f}\left(x_{1}\right)=B_{1} \sin \left(\frac{\Omega}{\kappa} x_{1}\right)+B_{2} \cos \left(\frac{\Omega}{\kappa} x_{1}\right) \text {, }
$$

where $A_{1}, A_{2}$ and $B_{1}, B_{2}$ are integration constants to be determined through the boundary conditions (BCs). The BCs for the system require continuity at the supported/unsupported transition:

$$
\begin{aligned}
& u_{s}(0)=u_{f}(0), \\
& u_{s}^{\prime}(0)=u_{f}^{\prime}(0),
\end{aligned}
$$

where prime stands for $x_{1}$ differentiation. Furthermore, consideration of the Floquet-Bloch waves lends the periodicity conditions

$$
\begin{aligned}
& u_{s}\left(-\frac{\alpha}{2}\right)=u_{f}\left(1-\frac{\alpha}{2}\right) \rho, \\
& u_{s}^{\prime}\left(-\frac{\alpha}{2}\right)=u_{f}^{\prime}\left(1-\frac{\alpha}{2}\right) \rho,
\end{aligned}
$$

where $\rho \neq 0$ is the characteristic multiplier. For a secondorder ODE, there are two nonnecessarily distinct characteristic multipliers, which are denoted by $\rho_{1}$ and $\rho_{2}$. Besides, let $\rho_{k}=\exp \left(m_{k}\right)$ with $k \in\{1,2\}$; then $m_{k}=i q$ is the characteristic exponent.

\section{Boundedness and Periodicity of the Solution}

Equation (5) is known as Hill's equation, after Hill who studied it in 1886 in the context of lunar dynamics [33]. For the sake of clarity, we shall write $(0, a)$ for the periodicity interval $(-\alpha / 2,1-\alpha / 2)$. It is easy to find a fundamental system of solutions $\phi_{1}\left(x_{1}\right), \phi_{2}\left(x_{1}\right)$ such that

$$
\begin{aligned}
& \phi_{1}(0)=1, \\
& \phi_{1}^{\prime}(0)=0, \\
& \phi_{2}(0)=0, \\
& \phi_{2}^{\prime}(0)=1 .
\end{aligned}
$$

This is a set of two particular linearly independent solutions of the ODE (6); see [9] for further details. It is well known that, for any second-order ODE,

$$
\begin{aligned}
W\left(\phi_{1}\left(x_{1}\right), \phi_{2}\left(x_{1}\right)\right)= & \operatorname{det}\left[\begin{array}{ll}
\phi_{1}\left(x_{1}\right) & \phi_{2}\left(x_{1}\right) \\
\phi_{1}^{\prime}\left(x_{1}\right) & \phi_{2}^{\prime}\left(x_{1}\right)
\end{array}\right] \\
= & \phi_{1}\left(x_{1}\right) \phi_{2}\left(x_{1}\right)^{\prime} \\
& -\phi_{2}\left(x_{1}\right) \phi_{1}^{\prime}\left(x_{1}\right) \equiv 1,
\end{aligned}
$$

where $W$ is the Wronskian of the fundamental solutions [34]. In light of the problem's symmetry, $\phi_{1}$ is an even and $\phi_{2}$ an odd function. Let us introduce the discriminant

$$
\begin{aligned}
D= & \phi_{1}(a)+\phi_{2}^{\prime}(a)=\rho_{1}+\rho_{2} \\
= & 2 f(\Omega) \sin \left[\frac{(1-\alpha) \Omega}{\kappa}\right] \sin \left(\frac{\alpha \sqrt{\Omega^{2}-1}}{\kappa}\right) \\
& +2 \cos \left[\frac{(1-\alpha) \Omega}{\kappa}\right] \cos \left(\frac{\alpha \sqrt{\Omega^{2}-1}}{\kappa}\right),
\end{aligned}
$$

being

$$
f(\Omega)=\frac{1-2 \Omega^{2}}{2 \Omega \sqrt{\Omega^{2}-1}}=-\frac{1-1 / 2 \Omega^{2}}{\sqrt{1-1 / \Omega^{2}}} .
$$

Hill's equation is said to be unstable if all nontrivial solutions are unbounded in $\mathbb{R}$ and conditionally stable if there is a 


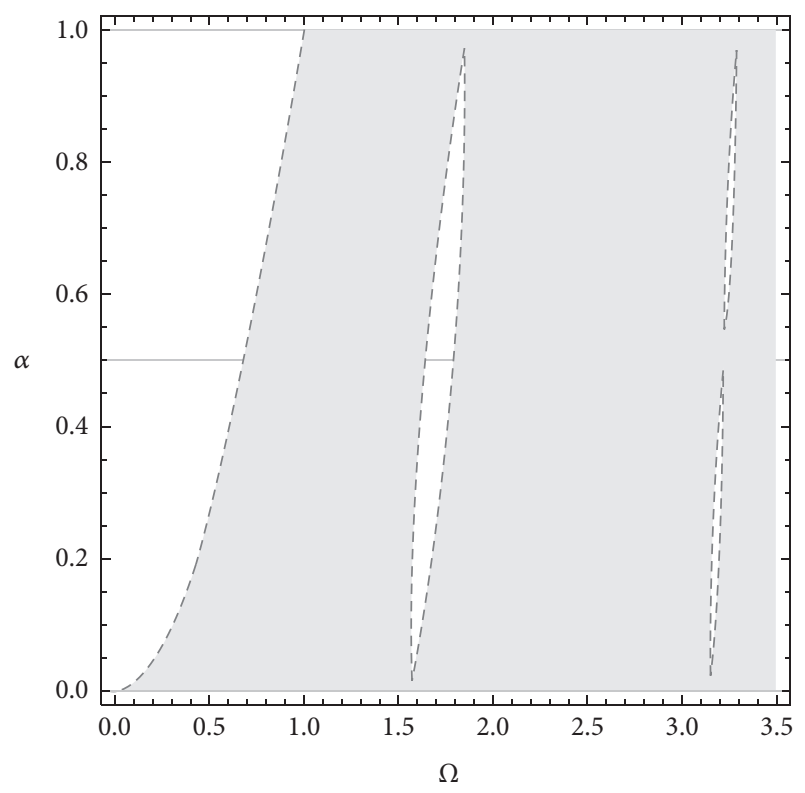

FIGURE 2: Stability regions (shaded in gray) for Hill's equation (5) at $\kappa=0.5$. Solid and dashed curves represent the level lines $D= \pm 2$, respectively.

nontrivial solution bounded in $\mathbb{R}$ and stable if all solutions are bounded in $\mathbb{R}$.

By Theorem 1.3.1 of [9], the solution is stable if $|D|<2$ and unstable when $|D|>2$ and special consideration is required for the case $|D|=2$. Indeed, when $|D|<2$, the characteristic multipliers $\rho_{1}$ and $\rho_{2}$ are complex conjugated and have unit modulus, whereupon the characteristic exponents are opposite; that is, $q_{1}=-q_{2}$. Figure 2 plots the bounding curves $D=2$ (dashed) and $D=-2$ (solid) as a function of $\Omega$ and $\alpha$ for $\kappa=0.5$. Stable regions are shaded in gray. It is seen that Band Gaps (BGs) are bounded by dashed and solid curves in succession. When $D= \pm 2$ it is $\rho_{1}=\rho_{2}= \pm 1$, respectively.

It is also well known [9, Section 1.2] that the bounding curve $D=2$ corresponds to solutions in the form

$$
\begin{aligned}
& \psi_{1}\left(x_{1}\right)=p_{1}\left(x_{1}\right), \\
& \psi_{2}\left(x_{1}\right)=p_{2}\left(x_{1}\right),
\end{aligned}
$$

where $p_{1}\left(x_{1}\right)$ and $p_{2}\left(x_{1}\right)$ are periodic functions with period 1 , provided that the following condition which grants the availability of two eigenvectors for the repeated eigenvalue $\rho_{1,2}=1$ holds

$$
\phi_{2}(a)=\phi_{1}^{\prime}(a)=0
$$

Under the same condition, the bounding curve $D=-2$ corresponds to solutions in the form

$$
\begin{aligned}
& \psi_{1}\left(x_{1}\right)=P_{1}\left(x_{1}\right), \\
& \psi_{2}\left(x_{1}\right)=P_{2}\left(x_{1}\right),
\end{aligned}
$$

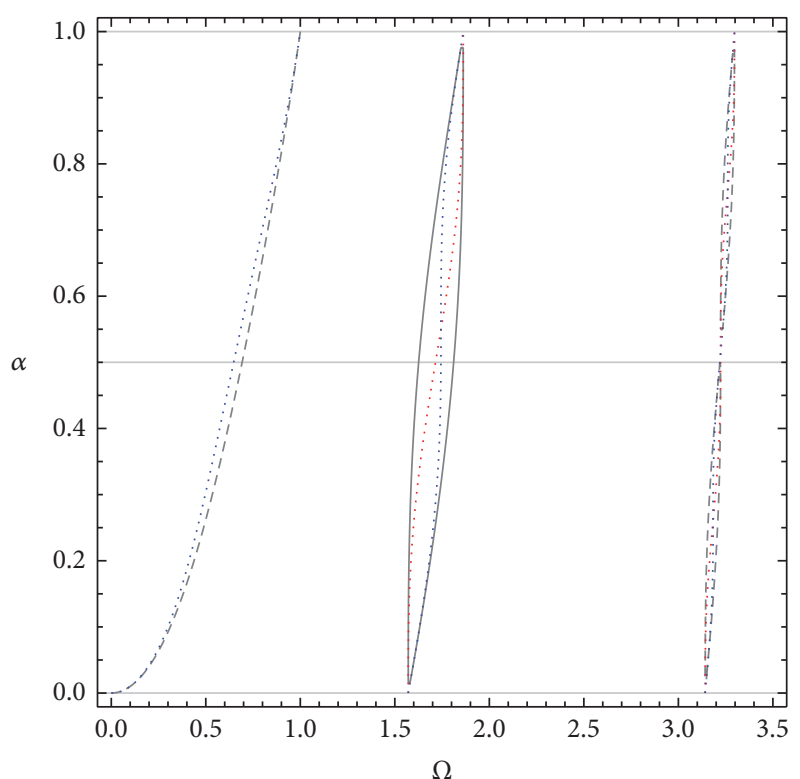

FIgURE 3: Periodic and semiperiodic solutions for Hill's equation (5) at $\kappa=0.5$. The red and the blue dotted curves represent the solution of the first and the second of the conditions (16), respectively.

where $P_{1}\left(x_{1}\right)$ and $P_{2}\left(x_{1}\right)$ are semiperiodic function with period 1; that is,

$$
P_{i}\left(x_{1}+1\right)=-P_{i}\left(x_{1}\right), \quad i=1,2 .
$$

In Figure 3, the solution curves for the first and the second of the conditions (16) are plotted in dotted line style and they partly overlie the conditionally stable curves $D= \pm 2$. However, it is observed that such conditions are satisfied only at some very special points. Indeed, we have

$$
\begin{aligned}
(1 & \left.-\Omega^{2}\right) \phi_{2}(a)-\kappa^{2} \phi_{1}^{\prime}(a) \\
& =\frac{\kappa}{\Omega} \sin \left(\frac{(1-\alpha) \Omega}{\kappa}\right) \cosh \left(\frac{\alpha \sqrt{1-\Omega^{2}}}{\kappa}\right),
\end{aligned}
$$

whence crossing is possible either when

$$
\Omega=n \frac{\kappa \pi}{1-\alpha}, \quad n \in\{0,1,2,3, \ldots\}
$$

or when $\alpha=1$. The case $\alpha=1$ relates to a fully supported string for which $\phi_{1}^{\prime}(a)$ and $\phi_{2}(a)$ are both proportional to

$$
\sqrt{\Omega^{2}-1} \sinh \frac{\sqrt{\Omega^{2}-1}}{\kappa},
$$

so that they vanish at the isolated points $\Omega=\sqrt{1+n^{2} \kappa^{2} \pi^{2}}$, $n \in\{0,1,2, \ldots\}$. The first point, $\Omega=1$, corresponds to the pivotal frequency for the 0th BG. Conversely, the case $\alpha=0$ corresponds to a free string, which is a nondispersive situation. Indeed, BGs collapse into single points which, according to (20), are located at $\Omega=n \kappa \pi$. Other $(\Omega, \alpha, \kappa)$ combinations exist which satisfy $|D|=2$ and (16), such as $(3.22313,0.512649,0.5)$. In all such points two periodic 
(or semiperiodic) solutions exist and conditional stability is reverted to stability. However, in the general case, (16) never hold together and thus conditional stability remains. Indeed, the second of the conditions (15) is replaced by

$$
\psi_{2}\left(x_{1}\right)=x_{1} p_{1}\left(x_{1}\right)+p_{2}\left(x_{1}\right)
$$

which is obviously unstable. By the same token, the second of the conditions (17) becomes

$$
\psi_{2}\left(x_{1}\right)=x_{1} P_{1}\left(x_{1}\right)+P_{2}\left(x_{1}\right) .
$$

Thus, the coexistence problem for period 1 is generally answered in the negative; that is, a single periodic (or semiperiodic) solution exists which is accompanied by a nonperiodic one. Besides, from a mechanical standpoint, it is observed that, in the general case, a periodic solution $p(x)$ is not acceptable on physical grounds, as it conveys a jump discontinuity at the boundary, unless

$$
p(0)=p(a) .
$$

Likewise, a semiperiodic solution $P(x)$ is not acceptable unless

$$
P(0)=-P(a)
$$

We now prove that such conditions can always be met. To this aim we now take advantage of the problem's symmetry and recall that a nontrivial even solution exists if and only if

$$
\begin{aligned}
\phi_{1}^{\prime}\left(\frac{a}{2}\right) & =0 \\
\text { or } \phi_{1}\left(\frac{a}{2}\right) & =0,
\end{aligned}
$$

respectively, are periodic and semiperiodic, whereas an odd solution stands if and only if

$$
\begin{aligned}
\phi_{2}^{\prime}\left(\frac{a}{2}\right) & =0 \\
\text { or } \phi_{2}\left(\frac{a}{2}\right) & =0,
\end{aligned}
$$

again for periodic and semiperiodic [9, Theorem 1.3.4]. Now, when $D=2$ we have the nontrivial periodic solution $p_{1}\left(x_{1}\right)$ and let us define

$$
p_{1+}\left(x_{1}\right)=\frac{p_{1}\left(x_{1}\right)+p_{1}\left(a-x_{1}\right)}{p_{1}(0)+p_{1}(a)} .
$$

By the periodicity of $p_{1}$, clearly $p_{1+}$ is proportional to the even part of $p_{1}$ and it is periodic. Besides,

$$
\begin{aligned}
p_{1+}(0) & =p_{1+}(a), \\
p_{1+}^{\prime}\left(\frac{a}{2}\right) & =0,
\end{aligned}
$$

which are, respectively, (24) and the first of the conditions (26). With the choice for the denominator in (28), it is also $p_{1+}(0)=1$ by which we conclude that $p_{1+} \equiv \phi_{1}$ extended in

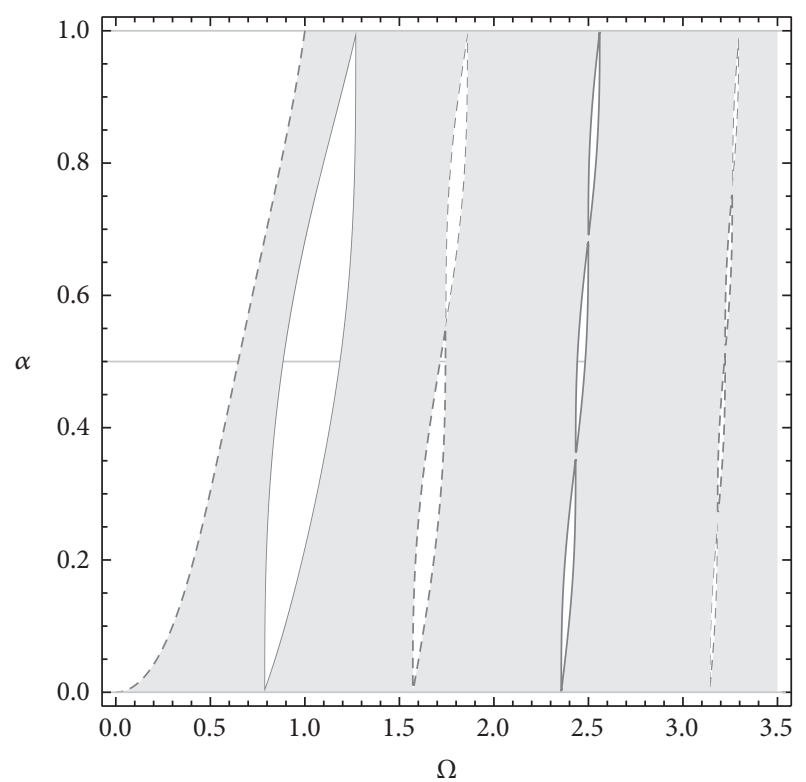

FIGURE 4: Stability regions (shaded in gray) for Hill's equation (5) at $\kappa=0.25$.

periodic fashion (it is also easy to show that no slope jump discontinuity is admitted by Hill's equation. Then, one can prove that $\phi_{1}^{\prime}(0)=\phi_{1}^{\prime}(a)=0$ and $\phi_{2}^{\prime}(0)= \pm \phi_{2}^{\prime}(a)$, where the sign is given in the periodic and semiperiodic situation, resp.). Likewise, when $D=-2$, we have the nontrivial semiperiodic solution $P_{1}\left(x_{1}\right)$ and let us define

$$
P_{1-}\left(x_{1}\right)=\frac{P_{1}\left(x_{1}\right)-P_{1}\left(a-x_{1}\right)}{P_{1}(0)-P_{1}(a)},
$$

which, in light of $P_{1}$ being semiperiodic, is again proportional to the even part of $P_{1}$. It is the sum of two semiperiodic functions; $P_{1-}(x)$ is semiperiodic; besides

$$
\begin{aligned}
P_{1-}(0) & =-P_{1-}(a), \\
P_{1-}\left(\frac{a}{2}\right) & =0,
\end{aligned}
$$

which are, respectively, (25) and the second of the conditions (27). With the choice for the denominator in (30), it is $P_{1-}(0)=1$ and we conclude that $P_{1-} \equiv \phi_{1}$ extended in semiperiodic fashion. Through the analogous definitions of $p_{1-}$ and $P_{1+}$, one gets the odd function $\phi_{2}$ extended in periodic and semiperiodic fashion, respectively.

The role of $\kappa$ is illustrated comparing Figure 2 with Figure 4 . It is seen that the frequency axis roughly scales with $\kappa$. Besides, reducing $\kappa$ brings wider BGs which tilt and tend to cluster together. It is noticed that no symmetry exists about $\alpha=0.5$.

\section{Dispersion Relation}

Imposing the BCs ((9), (10)) gives the dispersion relation:

$$
\frac{1}{2} D-\cos q=0
$$




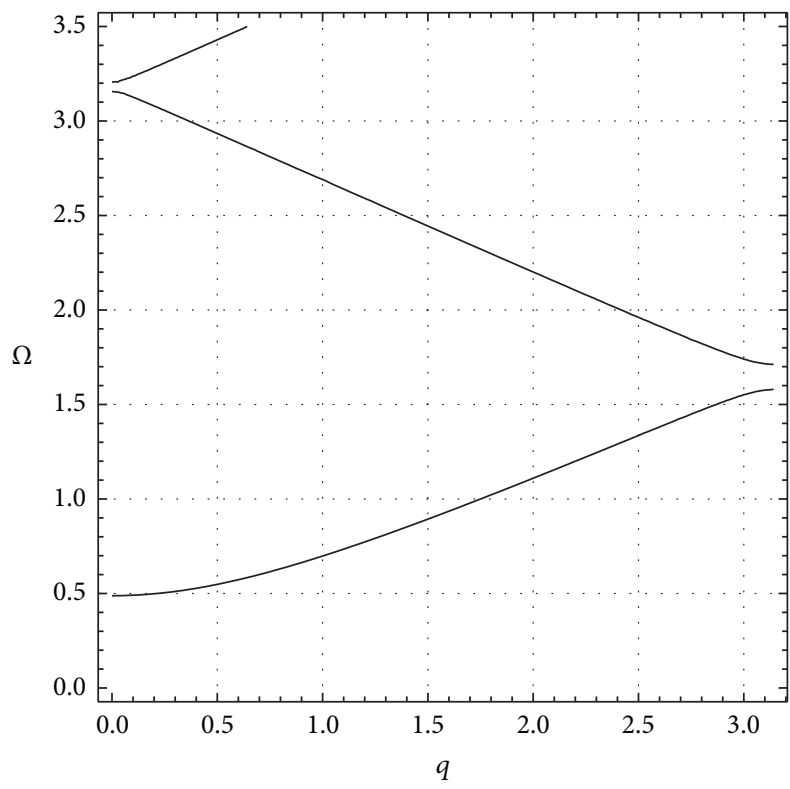

FIGURE 5: Dispersion relation for a string on a periodic support $(\kappa=$ $0.5, \alpha=0.25)$.

This relation can also be written as (see also [35])

$$
\begin{aligned}
\frac{1}{2}[1+ & f(\Omega)] \cos \left(\frac{(1-\alpha) \Omega-\alpha \sqrt{\Omega^{2}-1}}{\kappa}\right) \\
& +\frac{1}{2}(1-f(\Omega)) \cos \left(\frac{(1-\alpha) \Omega+\alpha \sqrt{\Omega^{2}-1}}{\kappa}\right) \\
& -\cos q=0
\end{aligned}
$$

and it is plotted in Figure 5 for $\kappa=0.5$ and $\alpha=0.25$. Equation (32) conforms to the form of (4) in [19].

It is easy to prove that $f(\Omega)$ quickly asymptotes -1 from below for $\Omega>1$, whence we can give a simple expression for the dispersion relation:

$$
\frac{(1-\alpha) \Omega+\alpha \sqrt{\Omega^{2}-1}}{\kappa}=q+k \pi, \quad k \in\{0,1,2, \ldots\} .
$$

Such approximation is superposed onto the dispersion curves in Figure 8 whereupon it is seen that it is very effective already at $\Omega$ close to 1 , although it is unable to capture the BGs.

The relevant bounding values for each band gap (BG) are obtained considering the periodic and the semiperiodic eigenvalue problems, for which two sets of BCs need to be considered, respectively:

(1) the periodicity conditions $u(0)=u(a)$ and $u^{\prime}(0)=$ $u^{\prime}(a)$

(2) the semiperiodicity conditions $u(0)=-u(a)$ and $u^{\prime}(0)=-u^{\prime}(a)$.

The relevant eigenfrequencies are denoted by $\lambda_{0}, \lambda_{1}, \ldots$ and $\mu_{0}, \mu_{1}, \ldots$, respectively, for the periodic and the semiperiodic eigenproblems. The eigenmode for the first periodic eigenfrequency $\lambda_{0}$ is shown in Figure 6 at $=0.5, \alpha=0.25$. Likewise,

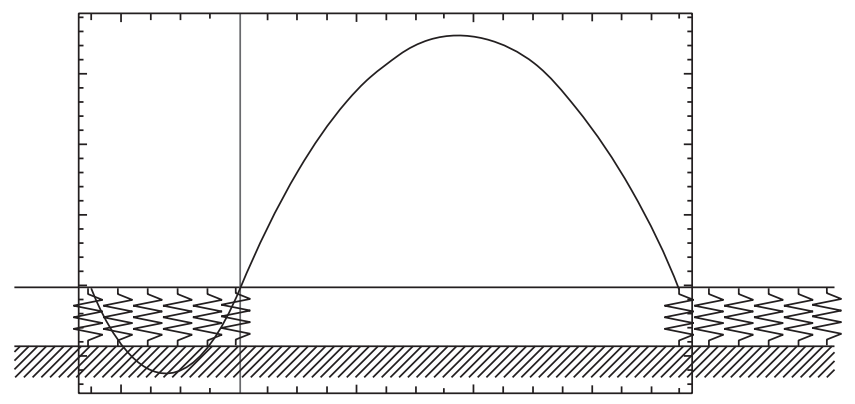

FIGURE 6: The first eigenmode, relative to the eigenfrequency $\lambda_{0}=$ 0.488445 at $\kappa=0.5, \alpha=0.25$, and $q=0$, is a periodic function.

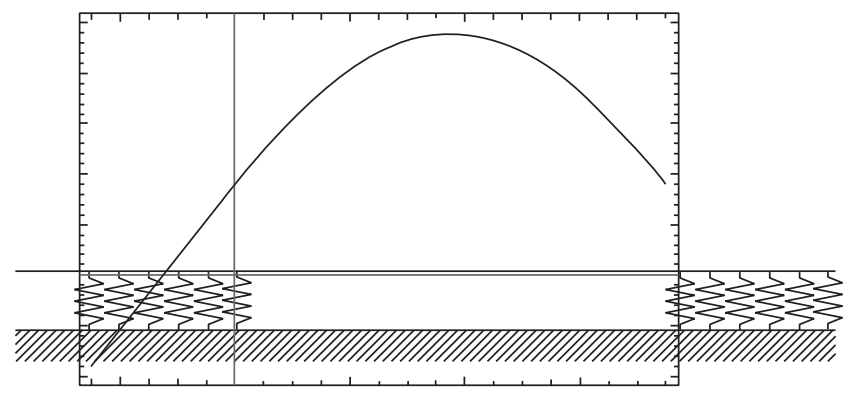

Figure 7: The eigenmode at the lower end of the first BG, relative to the eigenfrequency $\mu_{0}=1.57858$ at $\kappa=0.5, \alpha=0.25$, and $q=\pi$, is a semiperiodic function.

the eigenmode for the first semiperiodic eigenfrequency $\mu_{0}$ is shown in Figure 7. Such eigenfrequency is the lower boundary of the system's first BG.

The dispersion curve intersection with the axis $q=0$ is given by $\lambda_{m}$ while the intersection with the axis $q=\pi$ is given by $\mu_{m}$, where $m \in \mathbb{N}$. BGs' size is obtained by $\mu_{2 m}-\mu_{2 m+1}$ and $\lambda_{2 m+1}-\lambda_{2 m+2}$.

\section{Conclusions}

In this paper, the Floquet theory of periodic coefficient ODEs is applied to describe the behavior of a mechanical waveguide. A homogeneous elastic string periodically supported by an elastic Winkler foundation is considered and it is found that the governing equation is given by a second-order Hill equation. Floquet-Bloch periodic boundary conditions are enforced. The stability regions together with the dispersion relation are found in terms of the Floquet theory through the discriminant. An asymptotic approximation to the lowest cutoff frequency is given. The fundamental eigensolutions of the periodic and of the semiperiodic problem are also determined. It is remarked that the present methodology can be extended to tackle functionally graded beams [36-39] or plates $[40,41]$. Applications in the realm of civil engineering are also possible [28-32, 42]. A nice extension of the present theory could be related to temperature $[43,44]$ or viscoelastic effects in the fiber [45-48]. 


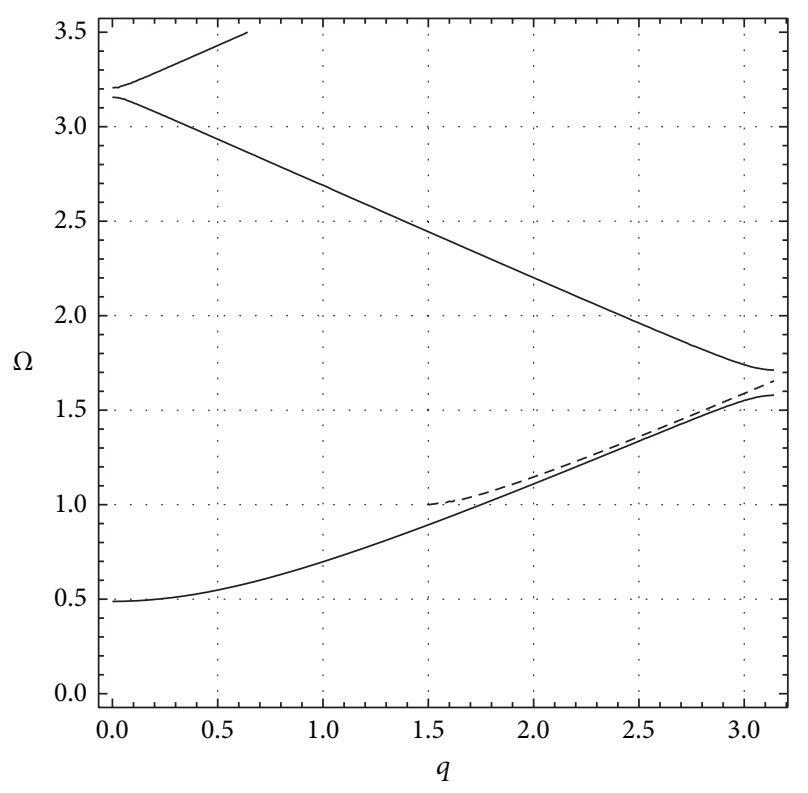

FIGURE 8: Dispersion relation (solid) superposed onto its first approximation (34) (dashed).

\section{Competing Interests}

The author declares that there are no competing interests.

\section{References}

[1] D. J. Mead, "Wave propagation and natural modes in periodic systems: I. Mono-coupled systems," Journal of Sound and Vibration, vol. 40, no. 1, pp. 1-18, 1975.

[2] D. J. Mead, "Wave propagation in continuous periodic structures: research contributions from Southampton, 1964-1995," Journal of Sound and Vibration, vol. 190, no. 3, pp. 495-524, 1996.

[3] D. Yu, Y. Liu, H. Zhao, G. Wang, and J. Qiu, "Flexural vibration band gaps in Euler-Bernoulli beams with locally resonant structures with two degrees of freedom," Physical Review B, vol. 73, no. 6, Article ID 064301, 2006.

[4] Y. Zhang, L. Han, and L.-H. Jiang, "Transverse vibration bandgaps in phononic-crystal Euler beams on a Winkler foundation studied by a modified transfer matrix method," Physica Status Solidi (B), vol. 250, no. 7, pp. 1439-1444, 2013.

[5] Y. Zhang, J. He, and L.-H. Jiang, "Flexural vibration band gaps characteristics in phononic crystal Euler beams on twoparameter foundation," Advances in Mechanical Engineering, vol. 5, Article ID 935258, 2013.

[6] J. Miklowitz, The Theory of Elastic Waves and Waveguides, Elsevier, New York, NY, USA, 2012.

[7] A. B. Movchan and L. I. Slepyan, "Resonant waves in elastic structured media: dynamic homogenisation versus Green's functions," International Journal of Solids and Structures, vol. 51, no. 13, pp. 2254-2260, 2014.

[8] A. Kudaibergenov, A. Nobili, and L. Prikazchikova, "On lowfrequency vibrations of a composite string with contrast properties for energy scavenging fabric devices," Journal of Mechanics of Materials and Structures, vol. 11, no. 3, pp. 231-243, 2016.

[9] M. S. Eastham, The Spectral Theory of Periodic Differential Equations, Scottish Academic Press, Edinburgh, UK, 1973.
[10] R. V. Craster, J. Kaplunov, and A. V. Pichugin, "High-frequency homogenization for periodic media," Proceedings of The Royal Society of London. Series A. Mathematical, Physical and Engineering Sciences, vol. 466, no. 2120, pp. 2341-2362, 2010.

[11] T. Antonakakis and R. V. Craster, "High-frequency asymptotics for microstructured thin elastic plates and platonics," Proceedings of the Royal Society A: Mathematical, Physical and Engineering Sciences, vol. 468, no. 2141, pp. 1408-1427, 2012.

[12] A. M. Tarantino, "On the finite motions generated by a mode I propagating crack," Journal of Elasticity, vol. 57, no. 2, pp. 85103, 1999.

[13] A. M. Tarantino, "Crack propagation in finite elastodynamics," Mathematics and Mechanics of Solids, vol. 10, no. 6, pp. 435-456, 2005.

[14] R. Luciano and J. R. Willis, "Hashin-Shtrikman based FE analysis of the elastic behaviour of finite random composite bodies," International Journal of Fracture, vol. 137, no. 1-4, pp. 261-273, 2006.

[15] A. Nobili, E. Radi, and L. Lanzoni, "A cracked infinite Kirchhoff plate supported by a two-parameter elastic foundation," Journal of the European Ceramic Society, vol. 34, no. 11, pp. 2737-2744, 2014.

[16] A. Caporale and R. Luciano, "Limit analysis of masonry arches with finite compressive strength and externally bonded reinforcement," Composites Part B: Engineering, vol. 43, no. 8, pp. 3131-3145, 2012.

[17] V. Salomoni, G. Mazzucco, C. Pellegrino, and C. Majorana, "Three-dimensional modelling of bond behaviour between concrete and FRP reinforcement," Engineering Computations, vol. 28, no. 1, pp. 5-29, 2011.

[18] A. Nobili, "Superposition principle for the tensionless contact of a beam resting on a Winkler or a Pasternak foundation," Journal of Engineering Mechanics, vol. 139, no. 10, pp. 1470-1478, 2013.

[19] D. J. Mead, "Free wave propagation in periodically supported, infinite beams," Journal of Sound and Vibration, vol. 11, no. 2, pp. 181-197, 1970.

[20] M. Gei, A. B. Movchan, and D. Bigoni, "Band-gap shift and defect-induced annihilation in prestressed elastic structures," Journal of Applied Physics, vol. 105, no. 6, Article ID 063507, 2009.

[21] G. Napoli and A. Nobili, "Mechanically induced HelfrichHurault effect in lamellar systems," Physical Review EStatistical, Nonlinear, and Soft Matter Physics, vol. 80, no. 3, Article ID 031710, 2009.

[22] S. Nemat-Nasser, J. R. Willis, A. Srivastava, and A. V. Amirkhizi, "Homogenization of periodic elastic composites and locally resonant sonic materials," Physical Review B, vol. 83, no. 10, Article ID 104103, 2011.

[23] R. V. Craster, J. Kaplunov, E. Nolde, and S. Guenneau, "Bloch dispersion and high frequency homogenization for separable doubly-periodic structures," Wave Motion, vol. 49, no. 2, pp. 333-346, 2012.

[24] J. Kaplunov and A. Nobili, "The edge waves on a Kirchhoff plate bilaterally supported by a two-parameter elastic foundation," Journal of Vibration and Control, 2015.

[25] A. Nobili and A. M. Tarantino, "Magnetostriction of a hard ferromagnetic and elastic thin-film structure," Mathematics and Mechanics of Solids, vol. 13, no. 2, pp. 95-123, 2008.

[26] V. Guidi, L. Lanzoni, and A. Mazzolari, "Patterning and modeling of mechanically bent silicon plates deformed through coactive stresses," Thin Solid Films, vol. 520, no. 3, pp. 1074-1079, 2011. 
[27] N. Tullini, A. Tralli, and L. Lanzoni, "Interfacial shear stress analysis of bar and thin film bonded to 2D elastic substrate using a coupled FE-BIE method," Finite Elements in Analysis and Design, vol. 55, pp. 42-51, 2012.

[28] A. M. Tarantino, "Homogeneous equilibrium configurations of a hyperelastic compressible cube under equitriaxial dead-load tractions," Journal of Elasticity, vol. 92, no. 3, pp. 227-254, 2008.

[29] V. A. Salomoni, C. E. Majorana, G. M. Giannuzzi, and A. Miliozzi, "Thermal-fluid flow within innovative heat storage concrete systems for solar power plants," International Journal of Numerical Methods for Heat and Fluid Flow, vol. 18, no. 7-8, pp. 969-999, 2008.

[30] V. A. Salomoni, C. E. Majorana, B. Pomaro, G. Xotta, and F. Gramegna, "Macroscale and mesoscale analysis of concrete as a multiphase material for biological shields against nuclear radiation," International Journal for Numerical and Analytical Methods in Geomechanics, vol. 38, no. 5, pp. 518-535, 2014.

[31] G. Dinelli, G. Belz, C. E. Majorana, and B. A. Schrefler, "Experimental investigation on the use of fly ash for lightweight precast structural elements," Materials and Structures, vol. 29, no. 194, pp. 632-638, 1996.

[32] P. Bisegna and R. Luciano, "Bounds on the overall properties of composites with debonded frictionless interfaces," Mechanics of Materials, vol. 28, no. 1-4, pp. 23-32, 1998.

[33] G. W. Hill, "On the part of the motion of the lunar perigee which is a function of the mean motions of the sun and moon," Acta Mathematica, vol. 8, no. 1, pp. 1-36, 1886.

[34] V. I. Smirnov, A Course of Higher Mathematics: Volume 2, Advanced Calculus, Pergamon Press, 1964.

[35] J. Kaplunov and A. Nobili, "Multi-parametric analysis of strongly inhomogeneous periodic waveguideswith internal cutoff frequencies," Mathematical Methods in the Applied Sciences, 2016.

[36] R. Luciano and J. R. Willis, "Non-local constitutive equations for functionally graded materials," Mechanics of Materials, vol. 36, no. 12, pp. 1195-1206, 2004.

[37] R. Barretta, L. Feo, and R. Luciano, "Some closed-form solutions of functionally graded beams undergoing nonuniform torsion," Composite Structures, vol. 123, pp. 132-136, 2015.

[38] R. Barretta, L. Feo, R. Luciano, and F. Marotti de Sciarra, "A gradient Eringen model for functionally graded nanorods," Composite Structures, vol. 131, pp. 1124-1131, 2015.

[39] R. Barretta, L. Feo, R. Luciano, and F. Marotti de Sciarra, "Variational formulations for functionally graded nonlocal Bernoulli-Euler nanobeams," Composite Structures, vol. 129, pp. 80-89, 2015.

[40] R. Barretta and R. Luciano, "Exact solutions of isotropic viscoelastic functionally graded Kirchhoff plates," Composite Structures, vol. 118, no. 1, pp. 448-454, 2014.

[41] A. Apuzzo, R. Barretta, and R. Luciano, "Some analytical solutions of functionally graded Kirchhoff plates," Composites Part B: Engineering, vol. 68, pp. 266-269, 2015.

[42] A. M. Tarantino, "On extreme thinning at the notch tip of a neoHookean sheet," The Quarterly Journal of Mechanics and Applied Mathematics, vol. 51, no. 2, pp. 179-190, 1998.

[43] G. Xotta, G. Mazzucco, V. A. Salomoni, C. E. Majorana, and K. J. Willam, "Composite behavior of concrete materials under high temperatures," International Journal of Solids and Structures, vol. 64, pp. 86-99, 2015.

[44] F. M. de Sciarra and M. Salerno, "On thermodynamic functions in thermoelasticity without energy dissipation," European Journal of Mechanics-A/Solids, vol. 46, pp. 84-95, 2014.
[45] R. Barretta, L. Feo, and R. Luciano, "Torsion of functionally graded nonlocal viscoelastic circular nanobeams," Composites Part B: Engineering, vol. 72, pp. 217-222, 2015.

[46] L. Dezi, G. Menditto, and A. M. Tarantino, "Homogeneous structures subjected to repeated structural system changes," Journal of Engineering Mechanics, vol. 116, no. 8, pp. 1723-1732, 1990.

[47] L. Dezi and A. M. Tarantino, "Time-dependent analysis of concrete structures with a variable structural system," $A C I$ Materials Journal, vol. 88, no. 3, pp. 320-324, 1991.

[48] L. Dezi, G. Menditto, and A. M. Tarantino, "Viscoelastic heterogeneous structures with variable structural system," Journal of Engineering Mechanics, vol. 119, no. 2, pp. 238-250, 1993. 


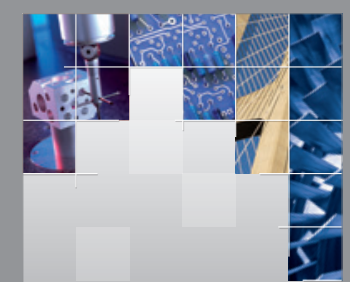

\section{Enfincering}
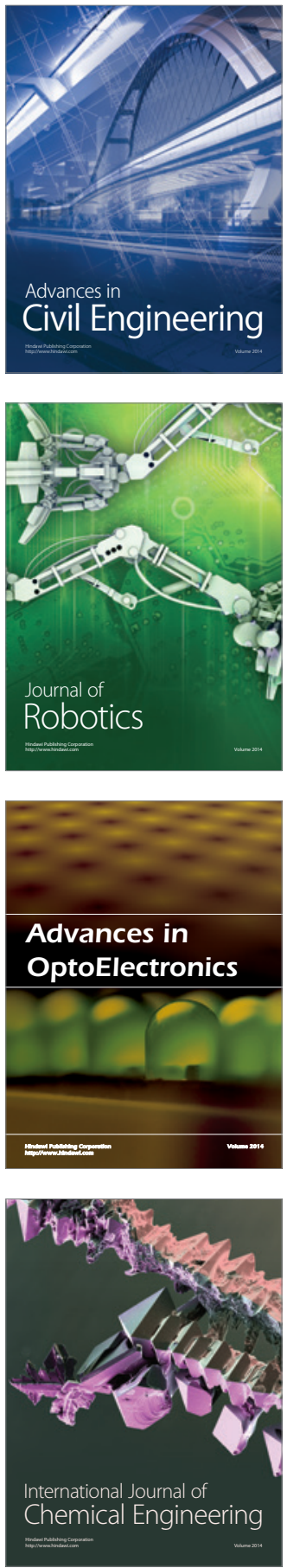

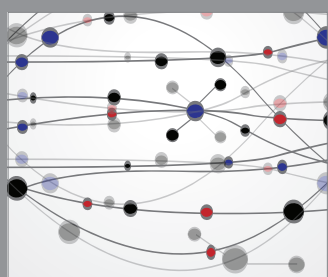

The Scientific World Journal

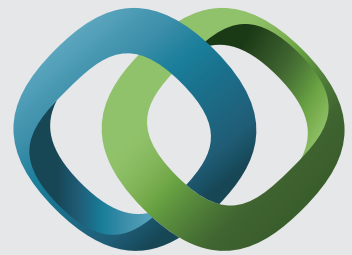

\section{Hindawi}

Submit your manuscripts at

http://www.hindawi.com
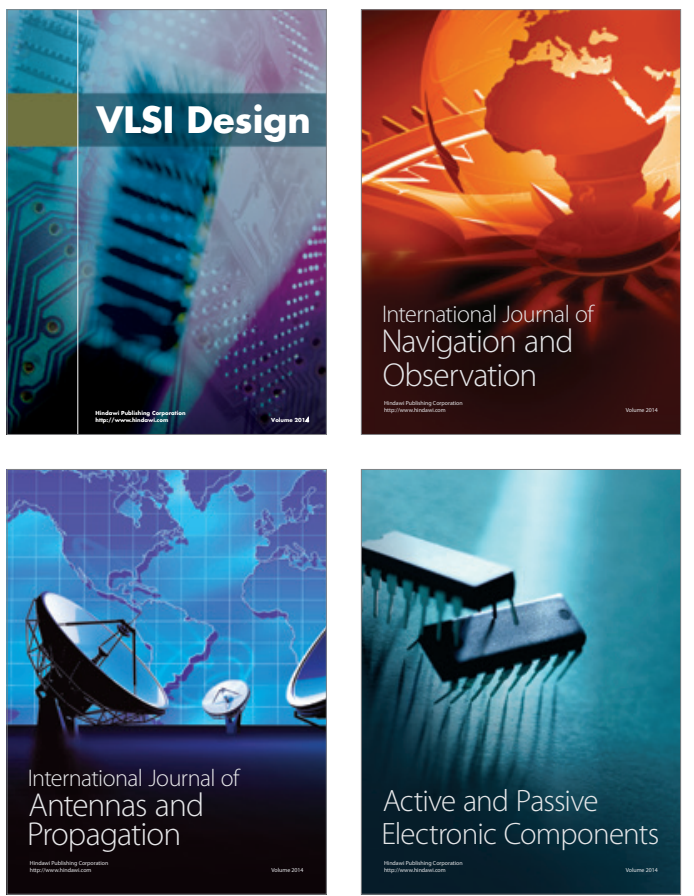
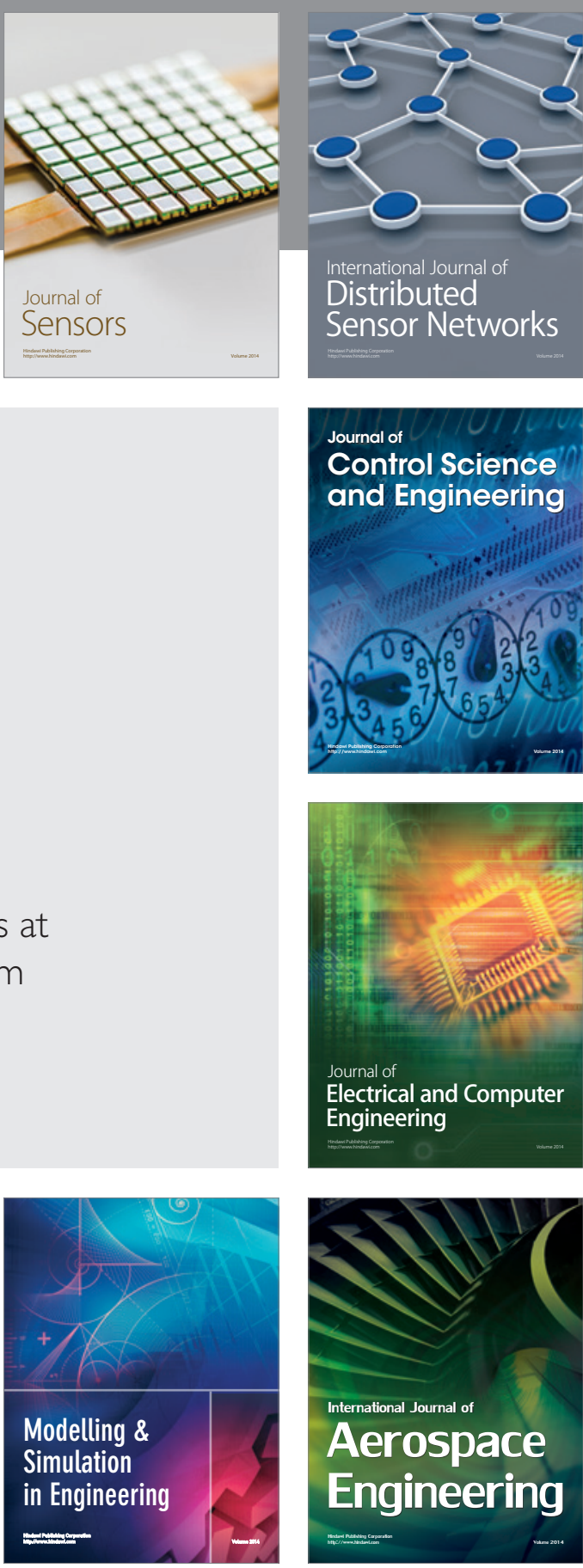

International Journal of

Distributed

Sensor Networks

Journal of

Control Science

and Engineering
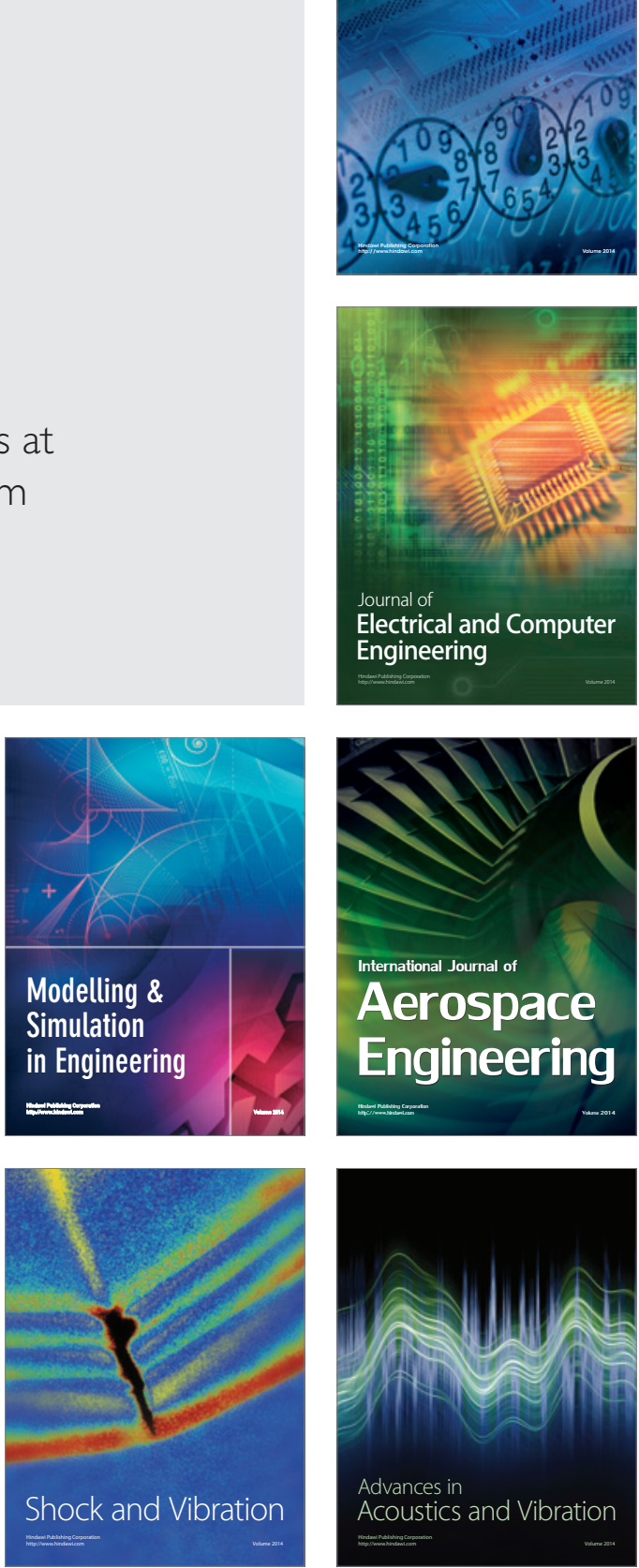